\section{Lesiones cutáneas asociadas a la humedad en geriatría: a propósito de tres casos}

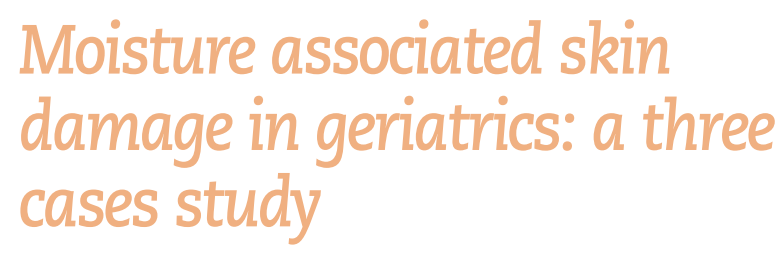

\author{
Raúl Muñoz Martín ${ }^{1, *}$ \\ Lorena Pulido Jiménez ${ }^{2}$ \\ Soraya García Peña ${ }^{3}$ \\ Genoveva Concepción Ortega Almeda ${ }^{4}$ \\ Ana Caro León ${ }^{5}$ \\ Rosa M. ${ }^{a}$ Gómez-Calcerrada Jiménez ${ }^{6}$ \\ Mercedes Corcobado Márquez?
}

1. Enfermero. Unidad de Cuidados Paliativos Domiciliarios. Hospital Provincial. Complejo Hospitalario de Toledo. Toledo. España.

2. Enfermera. Residencia de Personas Mayores "Ouijote y Sancho". Torrijos. Toledo. España.

3. Auxiliar de enfermería. Residencia de Personas Mayores "Quijote y Sancho". Torrijos. Toledo. España.

4. Auxiliar de enfermería. Área quirúrgica. Complejo Hospitalario de Toledo. Toledo. España.

5. Auxiliar de enfermería. Hospital General de Valdepeñas. Ciudad Real. España.

6. Enfermera. Centro de Salud Sillería. Toledo. España.

7. Enfermera. Gerencia de Salud de Cáceres. Cáceres. España.

*Autor para correspondencia.

Correo electrónico: ramuma@jccm.es (Raúl Muñoz Martín).

Recibido el 25 de abril de 2019; aceptado el 28 de junio de 2019.

\section{RESUMEN}

Las lesiones cutáneas asociadas a humedad son aquellas localizadas en la piel, presentadas como inflamación y/o erosión, causadas por exposición prolongada a fuentes de humedad con potencial irritativo. Los pacientes geriátricos, por cambios de estructura y resistencia de la piel, tienen más riesgo de padecerlas. Se presentan tres casos de pacientes geriátricos con estas lesiones. Exploración: Las pacientes presentan diversos tipos de lesiones: dermatitis intertriginosa, dermatitis asociada a la incontinencia y dermatitis asociada al exudado. Plan de actuación/

tratamiento: Se realizó un abordaje global e interdisciplinar, además

de local, incluyendo manejo de la humedad y uso de películas barrera siliconadas. Resultados: La evolución fue satisfactoria, con disminución o eliminación del eritema y la sintomatología asociada. Discusión: El abordaje global e interdisciplinar, y el uso de películas barrera siliconadas,

ha mejorado el confort y la evolución de las lesiones y reducido el tiempo de cura. Es preciso dar, a las lesiones por humedad en pacientes geriátricos, la relevancia que precisan.

PALABRAS CLAVE: Lesiones cutáneas asociadas a humedad, cuidados de la piel, geriatría, enfermería.

\section{ABSTRACT}

Moisture associated skin damage are those located on the skin, presented as inflammation and/or erosion, caused by prolonged exposure to moisture sources with irritative potential. Geriatric patients, due to structural changes and resistance of the skin, have a higher risk of suffering from them. Three cases of geriatric patients with these lesions are presented. Exploration: The patients present diverse types of injuries: intertriginous dermatitis, dermatitis associated with incontinence and dermatitis associated with exudate. Action/treatment plan: A global and interdisciplinary approach was carried out, in addition to local, including humidity management and use of silicone barrier films. Results: The evolution was satisfactory, diminishing or eliminating the erythema and the associated symptomatology. Discussion: The global and interdisciplinary approach, and the use of silicone barrier films, has improved comfort, the evolution of injuries and reduced the time of healing. It is necessary to give the relevance these injuries require in geriatric patients.

KEYWORDS: Moisture associated injuries, skin care, geriatrics, nursing.

\section{$\searrow$ INTRODUCCIÓN}

Las lesiones cutáneas asociadas a la humedad (LESCAH) son aquellas localizadas en la piel (no suelen afectar a tejidos subyacentes) que se presentan como una inflamación (eritema) y/o erosión de la misma, causada por la exposición prolongada (continua o casi continua) a diversas fuentes de humedad con potencial irritativo para la piel (p. ej., orina, heces, exudados de heridas, efluentes de estomas o fístulas, sudor, saliva o moco), lo que da lugar a su vez a diferentes tipos de LESCAH $^{1-3}$.
Las LESCAH han estado englobadas dentro de las úlceras por presión (UPP) hasta hace pocos años cuando, a raíz de los trabajos de Tom Defloor (2005), se las consideró una categoría aparte y se les comenzó a dar la importancia que requieren ${ }^{4}$.

Los cambios en la estructura y resistencia de la piel que presenta la población geriátrica van a favorecer, en este grupo de edad, la aparición de lesiones, entre ellas las LESCAH. A su vez, el aumento de comorbilidades que puede presentar esta población va a favorecer su mayor vulnerabilidad ante situaciones de estrés, aumentando su dependencia, y 
así el riesgo de lesiones, que van a repercutir muy negativamente a nivel general en los pacientes. Resulta imprescindible integrar unos cuidados de la piel adecuados 5,6 .

A su vez, a la hora de realizar cuidados de la piel, es preciso valorar los factores que pueden favorecer la aparición de estas lesiones, como la incontinencia, el mantenimiento de posturas prolongadas o el uso de materiales que favorezcan el acúmulo de humedad, para poder identificarlos, tratarlos y poder así llevar a cabo un plan de cuidados de la piel individualizado y continuado. Para llevar a cabo este abordaje, la colaboración de todo el equipo profesional que atiende a los pacientes es fundamental ${ }^{3}$.

Se presentan a continuación tres casos clínicos de pacientes geriátricos institucionalizados en residencia con presencia de LESCAH.

\section{CASO CLÍNICO 1}

\section{Historia clínica}

Mujer de 65 ańos. Presenta deterioro cognitivo tipo Alzheimer GDS 7 (Global Deterioration Scale o Escala de Deterioro Global de Reisberg), con agitación y mioclonías frecuentes, sin conexión con el medio ni habla. Alimentación por sonda de gastrostomía, encamada las 24 horas, biincontinente con uso de absorbentes, y episodios frecuentes de deposiciones líquidas.

Presenta una piel generalizada frágil, fina, con tendencia a los eritemas aun con presiones o roces leves. Presenta hiperhidrosis, más llamativa desde la colocación de la sonda de gastrostomía.

Ha presentado previamente diferentes episodios de dermatitis, que han sido tratadas de diversas maneras (corticoides, cremas hidratantes, uso de hamaca para favorecer la aireación, etc.), siendo todos ellos mínimamente eficaces.

\section{Exploración/valoración}

La paciente presenta lesiones dérmicas, principalmente eritemas intensos extensos en zonas de pliegues o de acúmulo de sudor, y con denudaciones de la piel, superficiales, en algunos de estos eritemas. La piel perilesional suele presentarse macerada figs. $1 \mathrm{~A} \mathrm{y} \mathrm{C)}$.

\section{Diagnóstico}

Podemos clasificar todas las lesiones como LESCAH. Se clasifican según propone el Grupo Nacional para el Estudio y Asesoramiento en Úlceras
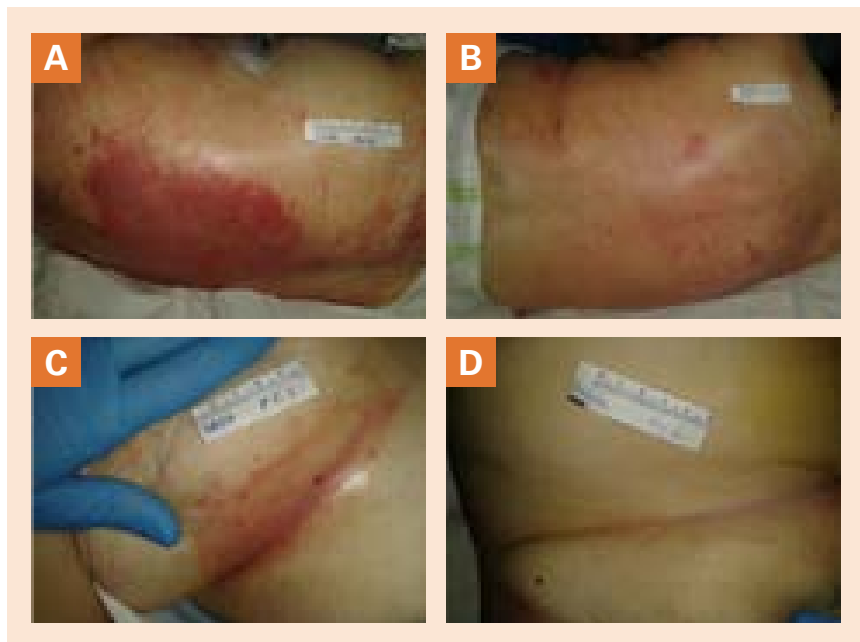

Figura 1. Lesiones por humedad en espalda y submamaria.

Por Presión y Heridas Crónicas (GNEAUPP)²:

- 1A: leve-moderado, piel íntegra de color rosado.

- 1B: intenso, piel íntegra de color rosa oscuro o rojo.

- 2A: leve-moderado, piel con erosión menor al 50\% del total del eritema.

- 2B: intenso, piel con erosión mayor al 50\% del total del eritema.

El diagnóstico, clasificación y evolución se exponen en la tabla 1.

\section{Plan de actuación/tratamiento}

Higiene diaria: se extremaron los cuidados al realizar la higiene diaria, poniendo especial interés en retirar la humedad excesiva de pliegues y de zonas afectadas. Se usaron cremas hidratantes en las zonas libres de lesión.

Retirada de sobrecolchón. Ropa de cama: se retiró el sobrecolchón para el manejo de la presión, para mejorar la aireación de la cama. La familia trajo sábanas propias, más suaves y con menor fricción que las del centro.

Cambios posturales. Se implementaron cambios posturales, de tal manera que se favoreciera la aireación de las zonas afectadas, especialmente la espalda, vigilando en todo caso la aparición de lesiones por presión, que no se produjeron.

Pauta de tratamiento local. Uso de productos barrera: el tratamiento local consistió en lo siguiente:

- En zonas con piel denudada: lavado con suero fisiológico, aplicación de espumas o apósitos siliconados, hasta el cierre de la lesión.

Tabla 1. Diagnóstico y evolución de las lesiones en el caso 1

\begin{tabular}{|c|c|c|c|c|}
\hline Zona & Agente causal probable & Diagnóstico & Categoría al inicio & Categoría posterior \\
\hline Perineo & Heces líquidas, orina & $\begin{array}{l}\text { Dermatitis asociada a la } \\
\text { incontinencia }\end{array}$ & $2 A$ & $\begin{array}{l}\text { 2A. Continúa presentando pequeñas zonas } \\
\text { de denudación, pero el eritema ha dismi- } \\
\text { nuido y presenta un color rosa pálido }\end{array}$ \\
\hline Pliegues submamarios & \multirow{4}{*}{ Sudor } & \multirow{4}{*}{ Dermatitis intertriginosa } & $1 \mathrm{~B}$ & \multirow{4}{*}{$\begin{array}{l}\text { 1A. El eritema ha disminuido en tamaño } \\
\text { y presenta un color rosa pálido }\end{array}$} \\
\hline Pliegue abdominal & & & $1 \mathrm{~B}$ & \\
\hline Cuello & & & $1 \mathrm{~B}$ & \\
\hline Espalda & & & $2 A$ & \\
\hline
\end{tabular}


- En zonas con eritema: aplicación una vez al día de crema barrera de polímeros (Cavilón Crema ${ }^{\circledR}$.

\section{Evolución}

La implementación de estas medidas surtió el efecto deseado, eliminando las zonas de piel denudada y reduciendo el eritema al cabo de 3 semanas. Estas medidas fueron más eficaces en las lesiones intertriginosas, siendo algo menor el efecto en la lesión por incontinencia perineal, en la que se produjeron denudaciones de la piel con mayor frecuencia (figs. 1 B y D y tabla 1).

\section{У CASO CLÍNICO 2}

\section{Historia clínica}

Mujer de 84 ańos. Presenta deterioro cognitivo de origen vascular GDS 4, con parkinsonismo, enfermedad pulmonar obstructiva crónica y fibrilación auricular anticoagulada. Presenta una ingesta adecuada, con deambulación autónoma e incontinencia ocasional con uso de absorbentes. Desorientación ocasional, mantiene conversación.

Tras un ingreso hospitalario por un posible accidente isquémico transitorio, la paciente regresó a la residencia con una UPP sacra de $25 \times 15 \mathrm{~cm}$ y categoría IV, y fractura de cadera, que requirió tratamiento quirúrgico.

Los cuidados a la paciente al alta se han centrado en su confort, cuidados de la fractura de cadera operada y cuidados de la UPP sacra. La paciente ha permanecido en cama 6 meses. Los productos usados para tratar la úlcera por presión incluían espumas con adhesivos acrílicos y productos barrera con base de óxido de cinc.

\section{Exploración/valoración}

La UPP se ha reducido considerablemente $(4 \times 6 \mathrm{~cm})$, pero presenta en la zona perilesional caudal enrojecimiento de la piel, con un tamańo de unos $4 \times 7 \mathrm{~cm}$, con algunas zonas erosionadas y maceradas (fig. $2 \mathrm{~A}$ ).

\section{Diagnóstico}

El diagnóstico, clasificación y evolución se exponen en la tabla 2.

\section{Plan de actuación/tratamiento}

Plan de higiene: se potencia la higiene perineal.

Pauta de tratamiento local. Uso de producto barrera: el centro solo dispone como película barrera de una crema infantil para las dermatitis de absorbentes, con un $10 \%$ de óxido de zinc, que es preciso renovar frecuentemente, y no permite adhesividad en la zona ni vigilancia, al taparla. En este caso, se retiró dicha crema y se comenzó a usar una película barrera de polímeros en espray (Silesse ${ }^{\circledR}$ ). Este producto presenta mayor facilidad de uso, permite una observación de la lesión al ser transparente y la adhesividad de los apósitos. A su vez, reduce el tiempo necesario para la cura, reduciendo la incomodidad de esta.

\section{Evolución}

Al cabo de 10 días, la lesión mejoró ostensiblemente, remitiendo casi en su totalidad (fig. 2 B y tabla 2).

\section{У CASO CLÍNICO 3}

\section{Historia clínica}

Mujer de 87 años. Presenta deterioro cognitivo moderado no filiado, con ictus menores de repetición. No orientada en espacio y tiempo, capaz de conversar. Buena ingesta, desplazamientos en silla de ruedas por inestabilidad en deambulación. Biincontinente, uso de absorbentes, con episodios frecuentes y diarios de deposiciones líquidas o semilíquidas.

\section{Exploración/valoración}

Presenta un gran eritema de color rojo intenso con algunas zonas de piel denudada en perineo que se extiende a cara interna de muslos. La paciente refiere, además, picor y molestias continuas en toda la zona (fig. $3 \mathrm{~A})$.

\section{Diagnóstico}

El diagnóstico, clasificación y evolución se exponen en la tabla 3.

\section{Plan de actuación/tratamiento}

Higiene perineal: se fomentó la higiene perineal, incrementando los cambios de absorbentes si era necesario. Se puso mayor énfasis en mantener la piel limpia y seca, especialmente en pliegues, cuidando de frotar lo menos posible y usando la cantidad mínima de jabón o productos jabonosos, a fin de evitar irritar la piel.

Pauta de tratamiento local: se cambió la crema disponible en la residencia por una crema de barrera de polímeros (Cavilón Crema ${ }^{\circledR}$ ). Se aplicaba una vez al día en todas las zonas afectadas.
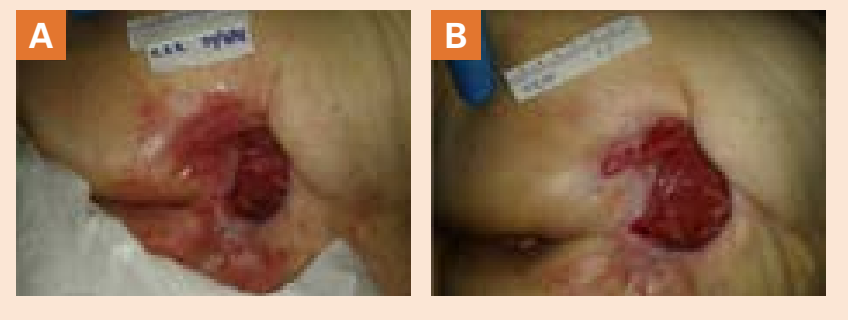

Figura 2. Lesión por humedad perilesional.

Tabla 2. Diagnóstico y evolución de las lesiones en el caso 2

\section{Zona}

Perilesional, zona caudal

\section{Agente causal probable}

Heces líquidas, orina, exudado de la herida

\section{Diagnóstico}

Dermatitis

perilesional/dermatitis por incontinencia
Categoría al inicio

Categoría posterior

1A. Remite casi completamente en eritema, presentando tan solo alguna zona de color rosa pálido 
Tabla 3. Diagnóstico y evolución de las lesiones en el caso 3

\begin{tabular}{|l|c|c|c|c|}
\hline Zona & Agente causal probable & Diagnóstico & Categoría al inicio & Categoría posterior \\
\hline $\begin{array}{l}\text { Perineo, cara interna de } \\
\text { muslos }\end{array}$ & Heces líquidas, orina & $\begin{array}{l}\text { Dermatitis por } \\
\text { incontinencia }\end{array}$ & $2 A$ & $\begin{array}{c}\text { 1A. Piel sin denudación, eritema } \\
\text { de color rosa más pálido }\end{array}$ \\
\hline
\end{tabular}
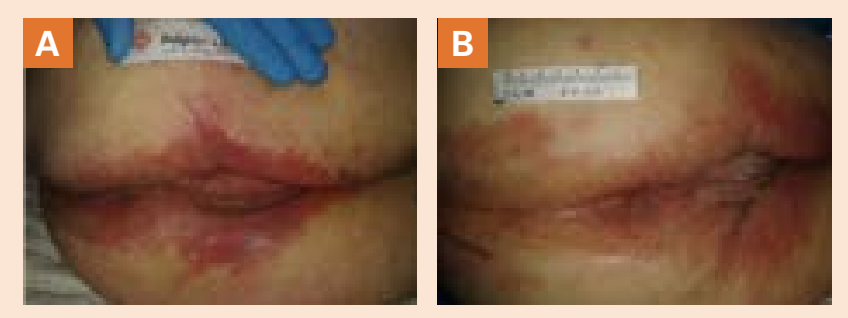

Figura 3. Dermatitis por incontinencia.

\section{Evolución}

A las 3 semanas, las zonas de piel denudada desaparecieron y, aunque el eritema se mantuvo en extensión, su color varió hacia un rojo-rosa más claro, refiriendo la paciente menores molestias como picor (figs. 3 B y tabla 3).

\section{У DISCUSIÓN}

Las lesiones por humedad siguen siendo muy poco conocidas en nuestro entorno. Es por ello por lo que los planes de tratamiento puedan ser muy dispares según el profesional que actúa, y no siempre son adecuados según el paciente y su lesión. La colaboración entre profesionales es imprescindible.
En los casos presentados se ha incidido mayormente en los cuidados de la piel, si bien es preciso señalar que los cuidados siempre han sido globales. Durante la atención prestada, se llevaron a cabo reuniones periódicas por parte del equipo de profesionales, en donde se estableció un plan de atención coordinado y consensuado, reevaluando de forma periódica las distintas actuaciones. Cabe recordar que el abordaje de cualquier paciente debe ser siempre global e interdisciplinar. La atención a la alimentación, sueño, temor o dolor resulta tan necesaria como el abordaje local.

Es precisa una adecuada formación de los profesionales para poder establecer, de forma consensuada y coordinada, planes de actuación adecuados y adaptados a la persona. En los casos presentados, quisiéramos remarcar el trabajo en equipo del personal del centro, que fue capaz de adaptar su actuación para atender las necesidades de cada paciente.

A su vez, el disponer de personal formado e implicado y de materiales adecuados resulta muy enriquecedor.

Los productos barrera de polímeros han resultado en nuestros casos más útiles que el producto disponible, al permitir vigilar la piel al ser transparentes, la adhesividad de los apósitos y, el formato en espray, un uso más fácil y rápido.

Esto ha supuesto un menor tiempo en la realización de las curas, un mejor mantenimiento de los apósitos, una más rápida evolución de las lesiones y una mejora en general del confort del paciente, lo que debe ser siempre nuestro principal objetivo como sanitarios

\section{Conflicto de intereses}

Los autores no declaran ningún conflicto de intereses.

\section{BIBLIOGRAFÍA}

1. Torra i Bou JE, Rodríguez Palma M, Soldevilla Ágreda JJ, García Fernández FP, Sarabia R, Zabala Blanco J, et al. Redefinición del concepto y del abordaje de las lesiones por humedad. Una propuesta conceptual y metodológica para mejorar el cuidado de las lesiones cutáneas asociadas a la humedad (LESCAH). Gerokomos. 2013;24(2):90-4.

2. García-Fernández FP, Soldevilla-Ágreda JJ, Pancorbo-Hidalgo $\mathrm{PL}$, Verdú-Soriano J, López-Casanova P, Rodríguez-Palma M. Clasificación-categorización de las lesiones relacionadas con la dependencia. Serie Documentos Técnicos GNEAUPP $n^{0}$ II. Grupo
Nacional para el Estudio y Asesoramiento en Úlceras por Presión y Heridas Crónicas. Logroño, 2014.

3. Rodríguez-Palma M, Pancorbo-Hidalgo PL, García-Fernández FP, Soldevilla-Ágreda JJ, Chiquero-Valenzuela S. Clasificación y diferenciación diagnóstica de las lesiones relacionadas con la dependencia. En: García-Fernández FP, Soldevilla-Ágreda JJ, Torra-Bou JE, eds. Atención Integral de las Heridas Crónicas. 2. ed. Logroño: GNEAUPP-FSJJ; 2016. p. 181-212.

4. Defloor T, Schoonhoven L, Fletcher J, Furtado K, Heyman H, Lubbers $M$, et al. Statement of the European Pressure Ulcer Advisory
Panel-pressure ulcer classification: differentiation between pressure ulcers and moisture lesions. J Wound Ostomy Continence Nurs. 2005;32(5):302-6;discussion 6.

5. Rodríguez Palma M. Revisión sistemática de los factores relacionados con la Dermatitis asociada a la Incontinencia. Propuesta de un nuevo modelo teórico [tesis doctoral en internet]. Alicante: Universidad de Alicante; 2015 [citada 09/05/2017]. 259 p. Disponible en: http://hdl.handle.net/10045/64610

6. Collier M, Simon D. Protecting vulnerable skin from moisture-associated skin damage. Br J Nurs. 2016;25(20):S26-32. 